\title{
Harmonic balance Navier-Stokes analysis of tidal stream turbine wave loads
}

\author{
A. Cavazzini, M.S. Campobasso, M. Marconcini, R. Pacciani, A. Arnone
}

\begin{abstract}
ARCTIC, a novel incompressible Reynolds-averaged Navier-Stokes finite volume code for the hydrodynamic analysis of open rotor unsteady loads is presented. One of its unique features is a harmonic balance solver enabling high-fidelity analyses of turbine periodic hydrodynamic loads with runtimes reduced by more than one order of magnitude over conventional time-domain CFD, and with negligible accuracy penalty. The strength of the new technology is demonstrated by analyzing with both harmonic balance and time-domain solvers the load fluctuations of a realistic tidal stream turbine. Such fluctuations are caused by a harmonic perturbation of the freestream velocity similar to that due to surface gravity waves.
\end{abstract}

KEYWORDS: Harmonic Balance, Navier-Stokes, artificial compressibility, tidal stream turbine, wave loads.

\section{Introduction}

Horizontal axis tidal stream turbines (TSTs) can be used to harvest renewable tidal stream energy, but their operation in harsh marine environments poses design challenges due to unsteady loads resulting in fatigue and/or extreme loads [1]. Loads due to current speed fluctuations resulting from surface gravity waves are predominantly periodic in several design-driving conditions, and their reliable prediction, at design stage, can be accomplished using Navier-Stokes Computational Fluid Dynamics (CFD). The time-domain $(T D)$ CFD solution of periodic rotor flows can be very

A. Cavazzini, M.S. Campobasso

Lancaster University, Department of Engineering, Gillow Avenue, Lancaster LA1 4YW, UK. email: a.cavazzini@lancaster.ac.uk, m.s.campobasso@lancaster.ac.uk

M. Marconcini, R. Pacciani, A. Arnone

University of Florence, Department of Industrial Engineering, Via Santa Marta 3, 50139 Florence, Italy.e-mail: michele.marconcini@unifi.it, roberto.pacciani@unifi.it, andrea.arnone@unifi.it 
time-consuming, whereas the frequency-domain harmonic balance $(H B)$ solution of the Reynolds-averaged Navier-Stokes (RANS) equations [7] of such flows can reduce runtimes by more than one order of magnitude with no significant accuracy loss, as demonstrated for the horizontal axis wind turbine case using compressible flow CFD [6]. To the best of the authors' knowledge, this study presents the first industrial-scale application of the $H B$ RANS technology to an incompressible periodic flow, namely the analysis of the unsteady hydrodynamic loads of a TST rotor subject to large harmonic variations of the oncoming current speed. The $T D$ and $H B$ governing equations are provided in Section 2; the numerical methods used by the new incompressible finite volume RANS code ARCTIC are in Section 3. The TST test case is analyzed in Section 4, and a summary is given in Section 5.

\section{Governing equations}

Turbulent incompressible flows can be determined by solving the RANS equations, featuring the Reynolds stress tensor in the momentum equations, and using Menter's Shear Stress Transport (SST) turbulence model for the turbulence closure. When solving rotor flow problems, it is convenient to formulate the governing equations in a rotating frame of reference, since, in the absence of external hydrodynamic forcing (e.g. yawed and/or sheared current) and instabilities (e.g. rotor blade dynamic stall), this enables solving a problem that is unsteady in the absolute (stationary) frame as a steady problem in the relative (rotating) frame. Using this approach, the grid position is fixed during the analysis. The unknown flow velocity components in ARCTIC are those at an arbitrary time in the absolute frame, even though the conservation laws are expressed in the rotating frame. The governing equations in the time- and frequency-domain are discussed in sections 2.1 and 2.2, respectively.

\subsection{Time-domain equations}

The Arbitrary Lagrangian-Eulerian (ALE) integral form of the system of the timedependent RANS and SST equations is written in a rotating Cartesian coordinate system, rotating about the $z$-axis with constant angular speed $\underline{\Omega}$, and with the $y$-axis usually being the blade axis. Given a control volume $C$ with boundary $S$, the $T D$ ALE integral form of the system of the RANS and SST equations is:

$$
\frac{\partial}{\partial t}\left(\int_{C} \mathbf{U}^{\prime} d C\right)+\oint_{S}\left(\underline{\Phi}_{c}-\underline{\Phi}_{d}\right) \cdot d \underline{S}-\int_{C} \mathbf{S}_{r} d C=0
$$

with $\mathbf{U}^{\prime}=\left[\begin{array}{llll}0 & \underline{\mathrm{u}}^{T} & k & \omega\end{array}\right]^{T}$. The symbols $\underline{\mathrm{u}}, k$, and $\omega$ denote, respectively, the flow velocity vector, the turbulent kinetic energy per unit mass, and the specific dissipation rate of $k$. The array of convective fluxes is: 


$$
\boldsymbol{\Phi}_{c}=\left[\begin{array}{c}
\left(\underline{\mathrm{u}}-\underline{\mathrm{u}}_{b}\right)^{T} \\
\left(\underline{\mathrm{u}}-\underline{\mathrm{u}}_{b}\right)^{T} \underline{\mathrm{u}}+p I \\
k\left(\underline{\mathrm{u}}^{\mathrm{u}}-\underline{\mathrm{u}}_{b}\right)^{T} \\
\omega\left(\underline{\mathrm{u}}-\underline{\mathrm{u}}_{b}\right)^{T}
\end{array}\right]
$$

where $I$ is the $(3 \times 3)$ identity matrix, and $p$ is the static pressure. The boundary velocity $\underline{\mathrm{u}}_{b}$ is given by:

$$
\underline{\mathrm{u}}_{b}=\underline{\Omega} \times \underline{\mathrm{r}}
$$

in which $\underline{\mathrm{r}}$ is the position vector. The definition of the generalized diffusive flux vector $\underline{\Phi}_{d}$ is reported in [3], and the source term $\mathbf{S}_{r}$ is given by:

$$
\mathbf{S}_{r}=\left[\begin{array}{llllll}
0 & -\Omega v & \Omega u & 0 & S_{k} & S_{\omega}
\end{array}\right]^{T}
$$

where $\Omega$ is the modulus of $\underline{\Omega}$. The symbols $S_{k}$ and $S_{\omega}$ denote respectively the source terms of the $k$ and $\omega$ equations of the SST turbulence model, and are defined in [3]. The equations of System (1) are nondimensionalized using the freestream density $\left(\rho_{\infty}\right)$, and absolute static pressure $\left(p_{\infty}\right)$, and a reference length $l_{r}$. Reference speed and time are respectively $v_{r}=\sqrt{p_{\infty} / \rho_{\infty}}$ and $t_{r}=l_{r} / v_{r}$.

\subsection{Harmonic balance equations}

The derivation of the frequency-domain $H B$ RANS and SST equations in a rotating frame follows the steps of that in the inertial frame [2,7], summarized below. The sought periodic flow field due to an external excitation of known angular frequency $\Omega_{e}$ is written as a truncated Fourier series with $\left(2 N_{H}+1\right)$ components, namely $N_{H}$ sinusoidal components, $N_{H}$ cosinusoidal components, and a time-independent term, with $N_{H}$ being a user-given parameter. The Fourier series expansion is then inserted in the $T D$ equations (System (1)). After suitable rearrangements [2], this results in the original system of $N_{p d e}=6$ time-dependent partial differential equations (PDEs) becoming a system of $\left[N_{p d e} \times\left(2 N_{H}+1\right)\right]$ time-independent PDEs, the solution of which yields the $\left(2 N_{H}+1\right)$ components of the truncated Fourier series. Writing the $H B$ RANS and turbulence model equations in the Fourier space, however, is challenging, due to the high level of nonlinearity of the equations involved; it was found more convenient to re-cast the $H B$ CFD equations in the TD [7]. Re-casting the $H B$ equations in the time-domain, results in the $H B$ equations becoming a system of $\left[N_{p d e} \times\left(2 N_{H}+1\right)\right]$ steady flow problems, and the $H B$ solution becoming a set of equally spaced flow states or snapshots of the unknown periodic flow. The $H B$ form of the RANS and SST equations is found to be:

$$
\Omega_{e} D\left(\int_{C_{H}} \mathbf{U}_{H}^{\prime} d C_{H}\right)+\oint_{S_{H}}\left(\underline{\Phi}_{c H}-\underline{\Phi}_{d H}\right) \cdot d \underline{S}_{H}-\int_{C_{H}} \mathbf{S}_{H} d C_{H}=0
$$


The array $\mathbf{U}_{H}^{\prime}$ is made up of $2 N_{H}+1$ periodic flow snapshots at $2 N_{H}+1$ equally spaced times $t_{n}$ given by:

$$
t_{n}=\frac{n}{\left(2 N_{H}+1\right)} \frac{2 \pi}{\Omega_{e}}, \quad n=0,1, \ldots, 2 N_{H}
$$

The structure of the array $\mathbf{U}_{H}$ is thus: $\mathbf{U}_{H}^{\prime}=\left[\tilde{\mathbf{U}}^{\prime}\left(t_{0}\right)^{T} \tilde{\mathbf{U}}^{\prime}\left(t_{1}\right)^{T} \ldots \tilde{\mathbf{U}}^{\prime}\left(t_{N_{H}}\right)^{T}\right]^{T}$, and is the same of that of all other variables with a subscript $H$ appearing in Eq. (5). The spectral operator $D$ is a $\left[\left(2 N_{H}+1\right) \times\left(2 N_{H}+1\right)\right]$ antisymmetric matrix $[2,6]$, which couples all $\left(2 N_{H}+1\right)$ flow snapshots.

\section{Numerical method}

The ARCTIC finite volume CFD code solves the $T D$ and $H B$ RANS and SST equations using an explicit multigrid algorithm similar to that of the compressible finite volume COSA code [3], based on Runge-Kutta (RK) pseudo-time-marching. The lack of a physical time-derivative in the continuity equation is dealt with by adopting Chorin's artificial compressibility [5], using which System (1) becomes:

$$
\Gamma^{-1} \frac{\partial}{\partial \tau}\left(\int_{C} \mathbf{U} d C\right)+\frac{\partial}{\partial t}\left(\int_{C} I^{*} \mathbf{U} d C\right)+\oint_{S}\left(\underline{\Phi}_{C}-\underline{\Phi}_{d}\right) \cdot d \underline{S}-\int_{C} \mathbf{S}_{r} d C=0
$$

where $\mathbf{U}=\left[\begin{array}{llll}p & \underline{\mathrm{u}}^{T} & k & \omega\end{array}\right]^{T}, \Gamma^{-1}=\operatorname{diag}\left(1 / \beta^{2}, 1,1,1\right), \beta$ is a user-given constant, $\tau$ is the pseudo-time, and $I^{*}$ is the $(4 \times 4)$ identity matrix with the first entry set to 0 .

\subsection{Space discretization}

ARCTIC solves the RANS and SST equations using structured multi-block grids. The convective fluxes per unit area $\Phi_{c f}$ along the cell face normal unit vector $\underline{\mathrm{n}}$ are computed using van Leer's second order MUSCL extrapolations and Roe's flux difference splitting, i.e. :

$$
\Phi_{c f}^{*}=\frac{1}{2}\left[\Phi_{c f}\left(\mathbf{U}_{L}\right)+\Phi_{c f}\left(\mathbf{U}_{R}\right)-\left|\frac{\partial \Phi_{c f}}{\partial \mathbf{U}}\right| \delta \mathbf{U}\right]
$$

where the superscript $*$, and the subscripts $f, L$ and $R$ denote respectively numerical approximation, face value, and values to the left and the right of the face, and $\delta \mathbf{U}=$ $\mathbf{U}_{R}-\mathbf{U}_{L}$. The term $\left|\partial \Phi_{c f} / \partial \mathbf{U}\right| \delta \mathbf{U}$ is the numerical dissipation, whose construction requires diagonalizing the Jacobian $\left(\partial \Phi_{c f} / \partial \mathbf{U}\right)$. Since, unlike in the case of the compressible RANS and SST equations [4], the Jacobians of the RANS and SST equations are decoupled in the incompressible case, only the Jacobian of the RANS equations is considered below, namely: 


$$
\frac{\partial \Phi_{c f}}{\partial \mathbf{U}}=\left[\begin{array}{cccc}
0 & \beta^{2} n_{x} & \beta^{2} n_{y} & \beta^{2} n_{z} \\
n_{x} u n_{x}+u_{n}-u_{b n} & u n_{y} & u n_{z} \\
n_{y} & v n_{x} & v n_{y}+u_{n}-u_{b n} & v n_{z} \\
n_{z} & u n_{z} & v n_{z} & w n_{z}+u_{n}-u_{b n}
\end{array}\right]
$$

where $u_{n}=u n_{x}+v n_{y}+w n_{z}$ and $u_{b n}=u_{b} n_{x}+v_{b} n_{y}+w_{b} n_{z}$ are, respectively, the fluid and the cell face velocity components along $\underline{\mathrm{n}}$.

The numerical dissipation of the RANS equations can be shown to be:

$$
\delta \Phi=\sum_{k=1}^{4}\left|\lambda_{k}\right| \delta \mathbf{W}_{k} R_{k}
$$

where

$$
\lambda_{1,2}=u_{n}-u_{b n} \quad \lambda_{3}=u_{n}-\frac{u_{b n}}{2}+c \quad \lambda_{4}=u_{n}-\frac{u_{b n}}{2}-c
$$

are the eigenvalues of $\left(\partial \Phi_{c f} / \partial \mathbf{U}\right), c=\sqrt{\beta^{2}+\left(u_{n}-u_{b n} / 2\right)^{2}}$ is the artificial speed of sound, $\delta \mathbf{W}_{k}=R^{-1} \delta \mathbf{U}$ is the variation of the characteristic variable associated with the eigenvalue $\lambda_{k}$, and $R_{k}$ and $R^{-1}$ are respectively the $k^{\text {th }}$ right eigenvector and the matrix of left eigenvectors of $\left(\partial \Phi_{c f} / \partial \mathbf{U}\right)$. The discretization of the diffusive fluxes and the source terms resulting from the production terms of the SST model is based on second order central differencing [3]. The implementation of the freestream boundary conditions is based on the characteristics of the Jabobian in Eq. (9), and the appropriate characteristics and freestream data to be used at inflow and outflow boundaries are selected automatically depending on whether the flow enters or leaves the domain. The cell face velocities given by Eq. (3) are computed using the freestream-capturing geometric formulation of [8].

\subsection{Numerical integration}

The $T D$ equations are solved using Jameson's dual-time-stepping. Denoting by $\mathbf{R}_{\Phi}$ the array of cell residuals resulting from the balance of the convective and diffusive fluxes over the cell faces, and all source terms over the cell volume, and $\mathbf{Q}$ the discrete counterpart of the array $\mathbf{U}$ evaluated at the cell center at physical time level $n+1$, and adopting a second order backward difference to discretize the physical time-derivative of $\mathbf{U}$, the semi-discrete counterpart of System (7) reads:

$$
\frac{\partial \mathbf{Q}^{n+1}}{\partial \tau} V+I^{*} \frac{3 \mathbf{Q}^{n+1}-4 \mathbf{Q}^{n}+\mathbf{Q}^{n-1}}{2 \Delta t} V+\mathbf{R}_{\Phi}\left(\mathbf{Q}^{n+1}\right)=0
$$

where $\mathbf{Q}^{n}$ and $\mathbf{Q}^{n-1}$ are the known solution arrays at time-levels $n$ and $n-1$ respectively, and $V$ is the cell volume. At each new time level $n+1, \mathbf{Q}^{n+1}$ is obtained by marching the equations in pseudo-time towards a steady state, discretizing the pseudo-time-derivative with an explicit four-stage RK scheme, and using local time-stepping, implicit residual smoothing (IRS) and multigrid for convergence 
acceleration. To avoid instabilities when the physical-time-step $\Delta t$ is notably smaller than the pseudo-time-step $\Delta \tau$, the term $3 \mathbf{Q}^{n+1} / 2 \Delta t$ in Eq. (12) is treated implicitly in the RK scheme. The $m^{\text {th }} T D$ RK cycle on the finest grid level is thus:

$$
\left[I+1.5 \alpha_{m} \frac{\Delta \tau}{\Delta t} I^{*}\right] \mathbf{Q}^{m}=\mathbf{Q}^{0}+1.5 \alpha_{m} \frac{\Delta \tau}{\Delta t} I^{*} \mathbf{Q}^{m-1}-\alpha_{m} \Delta \tau V^{-1} L_{I R S} \mathbf{R}_{g}\left(\mathbf{Q}^{m-1}\right)
$$

where $\alpha_{m}$ is the $m^{\text {th }}$ RK coefficient, $\mathbf{Q}^{m}$ is shorthand for $\mathbf{Q}^{n+1, m}, \mathbf{Q}^{0}$ is the solution at the beginning of the RK cycle, $L_{I R S}$ is the IRS operator, and

$$
\mathbf{R}_{g}\left(\mathbf{Q}^{m-1}\right)=I^{*} \frac{3 \mathbf{Q}^{n+1, m-1}-4 \mathbf{Q}^{n}+\mathbf{Q}^{n-1}}{2 \Delta t} V+\mathbf{R}_{\Phi}\left(\mathbf{Q}^{n+1, m-1}\right)
$$

Steady problems are solved with RK time-stepping enhanced by the aforementioned acceleration strategies, setting $\Delta \tau / \Delta t=0$ and $\mathbf{R}_{g}=\mathbf{R}_{\Phi}$ in Eq. (13). The smoother of Eq. (13) is for the RANS equations; additional terms appear in the smoother of the SST equations, due to the implicit treatment of the destruction terms [3].

The integration of the $H B$ equations is similar to that of steady problems. The $H B$ counterpart of the semi-discrete $T D$ Eq. (12) is:

$$
\frac{\partial \mathbf{Q}_{H}}{\partial \tau} V_{H}+D I_{H}^{*} \mathbf{Q}_{H} \Omega_{e} V_{H}+\mathbf{R}_{\Phi H}\left(\mathbf{Q}_{H}\right)=0
$$

in which the array $\mathbf{Q}_{H}$ has $\left(2 N_{H}+1\right)$ flow states, each referring to the physical times defined by Eq. (6), and thus has length $\left[N_{p d e} \times\left(2 N_{H}+1\right)\right]$. The array $\mathbf{R}_{\Phi H}$ has the same structure of $\mathbf{Q}_{H}$, and its $\left(2 N_{H}+1\right)$ states are the residuals associated with the convective fluxes, the diffusive fluxes and all source terms at the considered physical times, whereas the entries of $V_{H}$ are the cell volumes at the $\left(2 N_{H}+1\right)$ times. The $m^{\text {th }} H B$ RK cycle on the finest grid level reads:

$$
\mathbf{Q}_{H}^{m+1}=\mathbf{Q}_{H}^{0}+\alpha_{m} \Delta \tau_{H} V_{H}^{-1} L_{I R S, H} \mathbf{R}_{g H}\left(\mathbf{Q}_{H}^{m-1}\right) .
$$

where the entries of $\Delta \tau_{H}$ are the local time-steps for the $\left(2 N_{H}+1\right)$ flow states, $L_{I R S, H}$ is a block-matrix with $2 N_{H}+1$ blocks, each providing the IRS operator for one steady subproblem, and $\mathbf{R}_{g H}\left(\mathbf{Q}_{H}\right)=\Omega V_{H} D I_{H}^{*} \mathbf{Q}_{H}+\mathbf{R}_{\Phi H}\left(\mathbf{Q}_{H}\right)$.

\section{Results}

The test case considered below is an hypothetical two-blade TST with rotor diameter $D=20 \mathrm{~m}$ and hub at $40 \mathrm{~m}$ depth $\left(p_{\infty}=5 \mathrm{bar}\right.$ ), operating with current speed $w_{\infty}=2.5$ $\mathrm{m} / \mathrm{s}$. The tip-speed ratio is $\lambda=5.4$, and the blade geometry is that of the National Renewable Energy Laboratory Phase VI wind turbine used in a previous COSA compressible flow study [6]. The CFD structured multi-block grid used herein is the same one used in that COSA study, and has about $2.1 \mathrm{M}$ cells for a $180^{\circ}$ rotor sector. The nondimensionalized steady flow conditions analyzed herein are the same of the 
wind turbine in [6], with $\lambda=5.4$ and Reynolds number $R e=4.93 \times 10^{5}$, based on wind freestream velocity and $l_{r}=1 \mathrm{~m}$. This grid provided grid-independent solutions for the considered steady flow, with nondimensionalized minimum wall distance $y^{+}$of order 1 at all wall boundaries. To use this grid and the given $R e$ and $\lambda$ for the TST analysis herein, it was necessary to use a modified kinematic viscosity of $v=1.008 \times 10^{-5} \mathrm{~m}^{2} / \mathrm{s}$ and $l_{r}=1.988 \mathrm{~m}$. The dimensions of the physical domain, the boundary conditions (BCs) applied to its boundaries, and a view of the hydrofoil mesh are reported in Fig. 1. The TST steady flow and unsteady regime due to a harmonic fluctuation of the current speed, similar to that due to surface gravity waves, are examined in sections 4.1 and 4.2, respectively. The $180^{\circ}$ rotor sector was used in all cases enforcing periodicity BCs on the lateral boundaries.

\subsection{Steady flow analyses}

Here the simulations of the considered TST steady regime based on the new incompressible code ARCTIC and the well validated compressible code COSA [6] are cross-compared. To avoid compressibility effects, a freestream Mach number of 0.0206 and low-speed preconditioning (LSP) [4] are used in the COSA analysis. Denoting respectively by $F$ and $T$ rotor thrust and torque, the thrust coefficient $C_{F}$

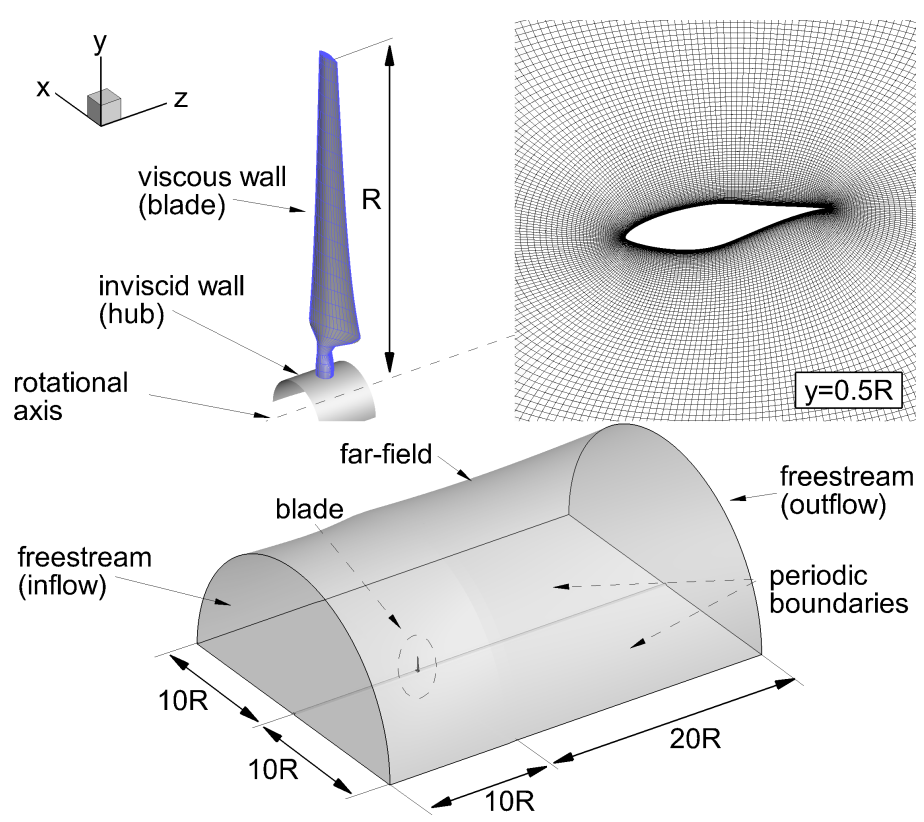

Fig. 1 TST blade geometry (top left), hydrofoil grid at $50 \%$ tip radius (top right), and domain dimensions and boundary conditions (bottom). 
and the torque coefficient $C_{T}$ are:

$$
C_{F}=\frac{8 F}{\rho_{\infty} w_{\infty}^{2} \pi D^{2}} \quad C_{T}=\frac{16 T}{\rho_{\infty} w_{\infty}^{2} \pi D^{3}}
$$

The pressure and viscous components of $C_{F}$ and $C_{T}$ computed by ARCTIC and COSA are compared in Tab. 1. An excellent agreement is noted, as the maximum difference between corresponding coefficients is lower than 2 percent.

Table 1 Pressure and viscous components of rotor thrust coefficient $C_{F}$ and torque coefficient $C_{T}$ computed by COSA compressible code and ARCTIC incompressible code.

\begin{tabular}{lllll}
\hline & $C_{F_{p}}$ & $C_{F_{v}}$ & $C_{T_{p}}$ & $C_{T_{v}}$ \\
\hline COSA & $7.54 \times 10^{-1}$ & $3.96 \times 10^{-3}$ & $9.66 \times 10^{-2}$ & $-1.52 \times 10^{-2}$ \\
ARCTIC & $7.45 \times 10^{-1}$ & $3.88 \times 10^{-3}$ & $9.48 \times 10^{-2}$ & $-1.52 \times 10^{-2}$ \\
\hline
\end{tabular}

The ARCTIC and COSA contours of velocity magnitude are compared in Fig. 2, where the left and right plots refer respectively to the blade sections at 30 and 80 percent rotor radius, and the velocity magnitude is normalized by the freestream value. An excellent agreement between the compressible and incompressible code solutions is observed also at this level, confirming the correctness of the implementation of the steady solver of the new incompressible code.

Figure 3 reports the convergence of the residuals of the continuity and momentum equations of COSA (left) and ARCTIC (right). The variable $\Delta l_{r}$ on the vertical axes is the logarithm in base 10 of the current residual normalized by the residual at the first iteration. No MG was used for these analyses, and the variable it on the $x$-axis is the number of single-grid iterations. Overall, the residual convergence of corresponding equations is comparable for the two codes, although the residual of the continuity equation of ARCTIC decreases by more than one order of magnitude
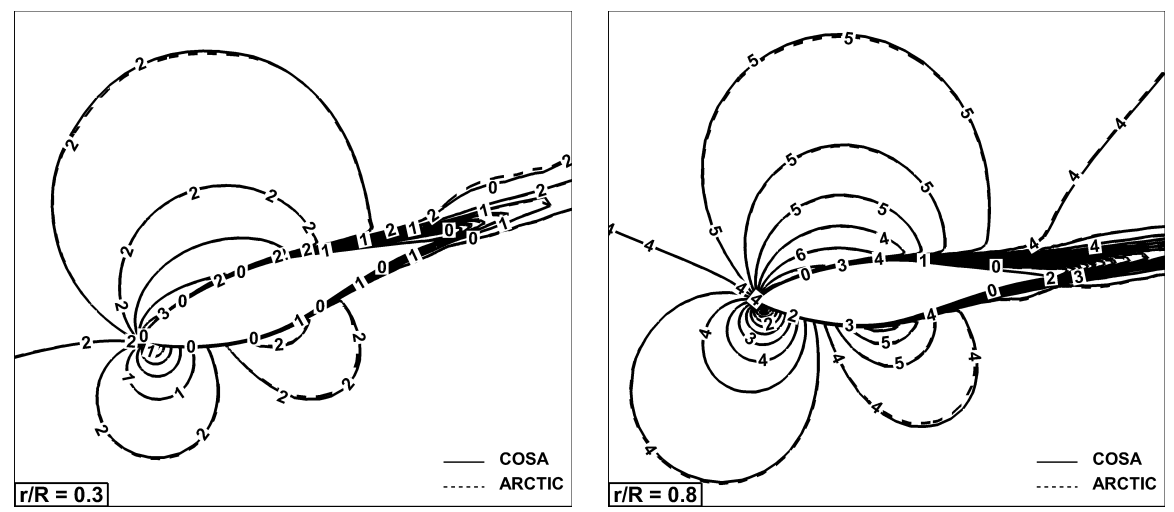

Fig. 2 Contours of velocity magnitude at 30 percent (left) and 80 percent (right) rotor radius computed by COSA and ARCTIC steady flow analyses. 
over that of COSA, whereas the final residuals of the momentum equations of COSA are marginally lower than those of ARCTIC.

\subsection{Unsteady flow due harmonic perturbation of current speed}

Here the unsteady periodic response of TST rotor loads to a harmonic planar perturbation of the freestream current speed is considered. The harmonic perturbation is prescribed as a harmonic variation of the grid velocity along the turbine axis using constant freestream conditions. This is to avoid the high grid refinement upstream of the rotor that would be required to prevent numerical dissipation from damping the propagation of free-stream perturbations. The longitudinal grid motion is:

$$
w_{g}(t)=\Delta w_{g} \cos \left(\Omega_{e} t\right)
$$

In the analyses below, $\Delta w_{g}=0.4 w_{\infty}, \Omega_{e}=\Omega$, and the mean flow has the same $\lambda$ and $R e$ of the steady regime examined in the previous section. The left plot of Fig. 4 depicts the profile of the rotor thrust coefficient $C_{F}$ over one oscillation period, obtained with $T D$ COSA and ARCTIC simulations, and $H B$ ARCTIC analyses using $N_{H}=1,3$; the right plot reports instead the periodic profiles of the rotor torque coefficient $C_{T}$ obtained with the same simulations. The peak $C_{T}$ higher than the theoretical maximum of 1 is merely due to using the mean rather than the instantaneous peak kinetic energy of the current. The TD analyses used 360 timesteps per period and $500 \mathrm{RK}$ cycles (no MG was used) per time-step. Thrust and torque were used to monitor the periodicity error. The $T D$ solutions were taken to be periodic once, for both outputs, the maximum difference between the instantaneous values of the last two periods was less than 0.5 percent of the maximum value of the last period, and this happened after 6 periods. The peaks of both $C_{F}$ and $C_{T}$
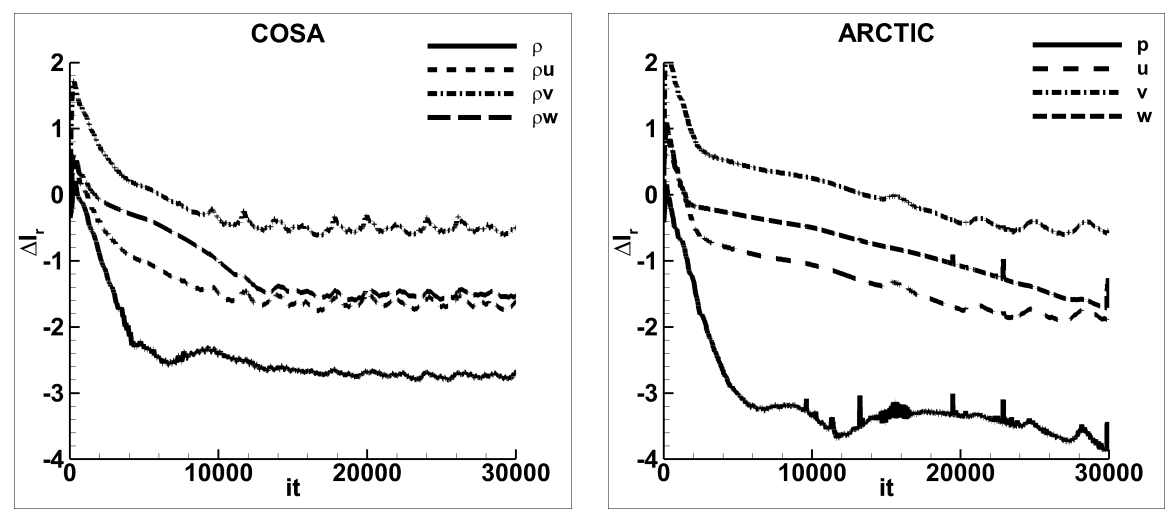

Fig. 3 Continuity and momentum residual convergence histories of steady COSA (left) and ARCTIC (right) solvers. 

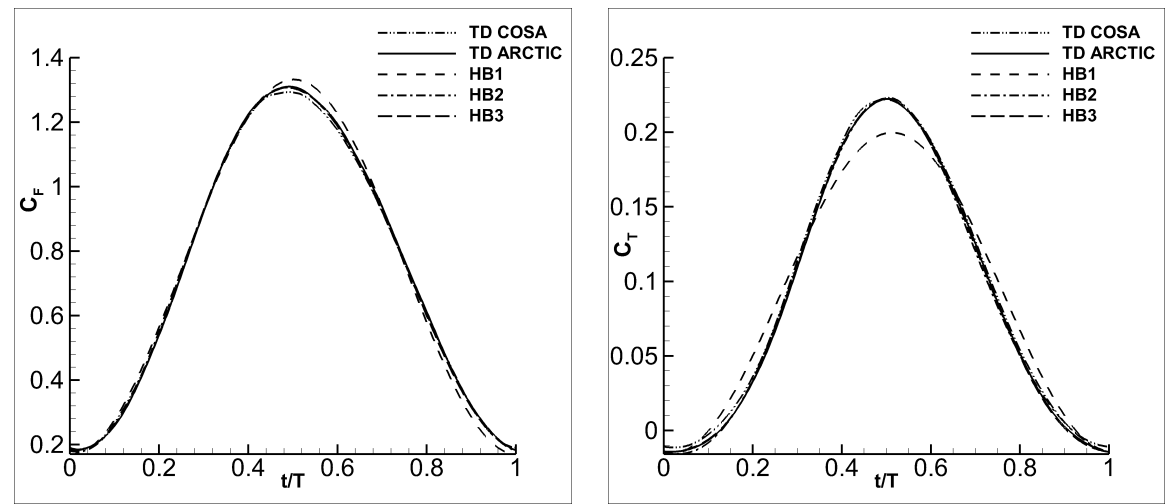

Fig. 4 Periodic profiles of thrust coefficient $C_{F}$ and torque coefficient $C_{T}$ obtained with $T D$ COSA and ARCTIC simulations, and $H B$ ARCTIC simulations.

in Fig. 4 are achieved at mid-period because the relative speed of the oncoming flow, resulting by subtracting the negative value of $w_{g}$ at mid-period (see Eq. (18)) from $w_{\infty}$, is maximum at this time. This yields close-to-maximum angle of attack and relative speeds along the blade, resulting in the aforementioned peaks. The curves of Fig. 4 show a very good agreement between the TD COSA and ARCTIC solutions, which validates the TD ARCTIC solver at the integral output level. Small differences are visible only around the peak region of the $C_{F}$ profiles, possibly due to lower residual convergence of COSA in this region. One also notes that $a$ ) the $H B$ ARCTIC profiles of $C_{F}$ and $C_{T}$ for $N_{H}=2$ (profiles labeled $H B$ 2) differ negligibly from the $T D$ ARCTIC profiles, and $b$ ) the use of more harmonics does not bring further solution improvement, as the $N_{H} 2$ and $N_{H} 3$ profiles are superimposed. The harmonic fluctuation of 40 percent of the current speed results in periodic fluctuations of about 70 and 175 percent of thrust and torque respectively, indicating power peaks more than twice as high as the steady power. Turbine control or safety system, however, would reduce these fluctuations or shut down the turbine.

The flow detail resolution of the $T D$ low-speed compressible and incompressible solvers, and the $H B 2$ incompressible analysis is examined in Fig. 5, comparing the three predictions of the contours of normalized velocity magnitude of the blade sections at 30 and 80 percent of rotor radius at 60 percent of the period. The $T D$ COSA and ARCTIC solutions differ very little, and no visible difference between the $T D$ and $H B 2$ ARCTIC solutions is noted.

The residual convergence histories of the continuity and momentum equations of the TD COSA and ARCTIC solvers at 60 percent of the period are reported in the left plot of Fig. 6. The residuals of the continuity and the $y$-momentum equations of the compressible solver stagnate after the first few iterations. The underlying lack of complete convergence may account for the small differences between the COSA and ARCTIC TD solutions observed above. The residual converge histories of the steady and $H B 2$ ARCTIC analyses are very similar, as seen in the right plot of Fig. 6, possibly due to the level of unsteadiness being insufficiently high to yield significant 

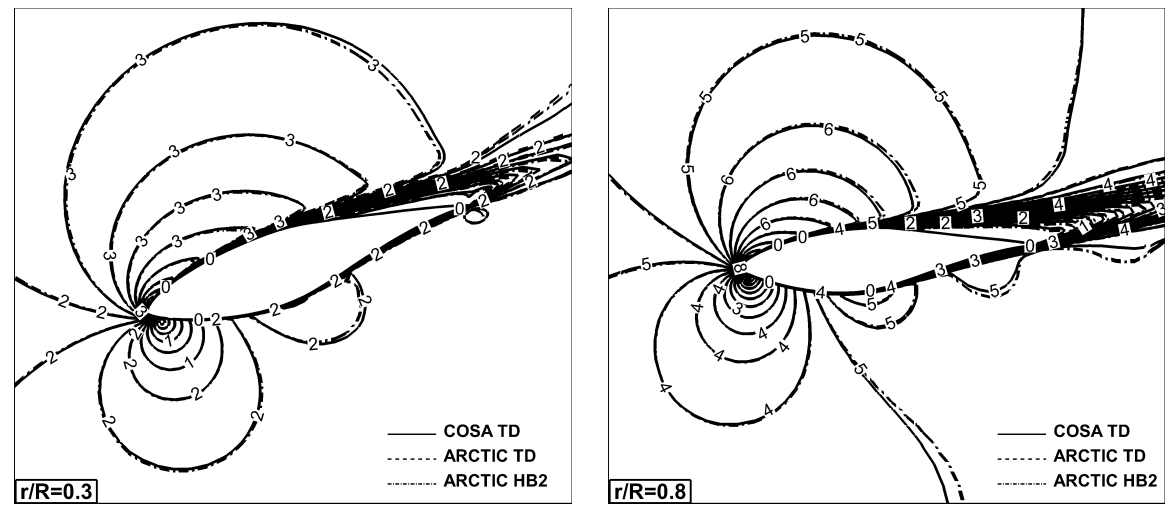

Fig. 5 Contours of velocity magnitude at 30 percent (left) and 80 percent (right) rotor radius at 60 percent oscillation period computed by $T D$ COSA, $T D$ ARCTIC, and $H B 2$ ARCTIC analyses.
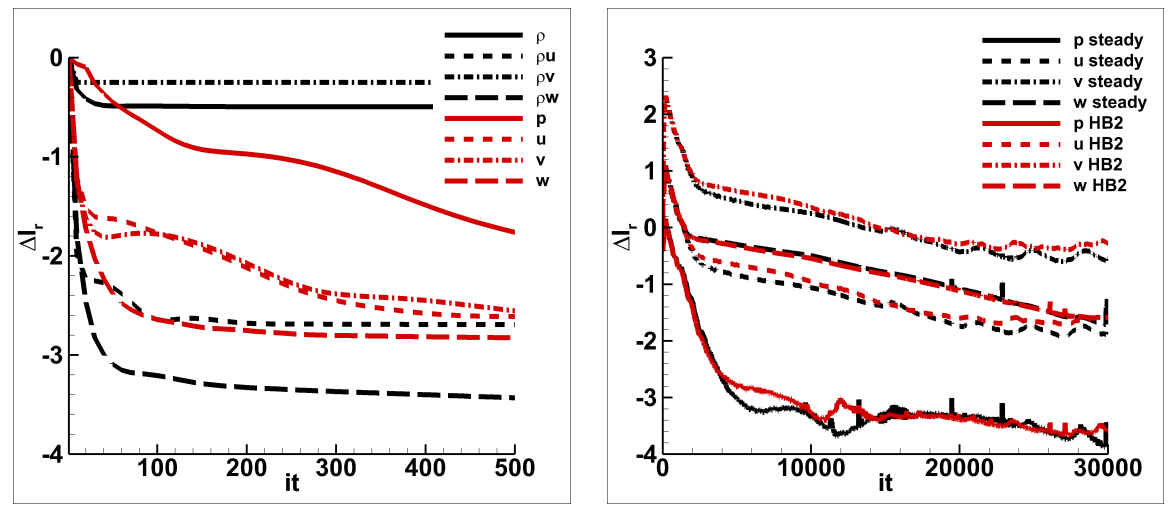

Fig. 6 Continuity and momentum residual convergence histories of $T D$ COSA and ARCTIC at 60 of oscillation period (left), and steady and $H B 2$ ARCTIC analyses (right).

differences between the spectral radii of the $H B$ and steady iterative solvers [2]. Table 2 provides the runtimes of the $T D$ COSA, $T D$ ARCTIC AND $H B$ ARCTIC analyses in terms of work-units, where one work-unit is the CPU-time needed for 4,000 RK cycles of the steady ARCTIC solver. One sees that $a$ ) the ARCTIC $H B 2$ solution, which differs negligibly from its $T D$ counterpart, is about $9.5 x$ faster than the $T D$ analysis, and $b$ ) the incompressible $T D$ analysis is 85 percent faster than the compressible $T D$ analysis, as COSA solves one more equation (that for energy) and performs additional operations for LSP.

Table 2 Computational cost in work-units of $T D$ COSA, $T D$ ARCTIC and $H B$ ARCTIC analyses.

\begin{tabular}{lllll}
\hline$T D$ COSA & $T D$ ARCTIC & $H B 1$ & $H B 2$ & $H B 3$ \\
\hline 752 & 405 & 25 & 43 & 60 \\
\hline
\end{tabular}




\section{Conclusions}

ARCTIC, an incompressible RANS code using Chorin's artificial compressibility was presented. The code has a conventional $T D$ solver and a novel frequencydomain $H B$ solver. Code validation relied on simulating with ARCTIC and COSA, a well validated LSP-enhanced compressible code, a steady flow condition of a two-blade TST and a periodic flow regime due to a planar harmonic perturbation of the oncoming current. Excellent agreement of blade loads and flow detail was found. Use of the incompressible $H B$ solver rather than its $T D$ counterpart enabled the CFD analysis of TST periodic hydrodynamic loads to be accelerated by more than 6 times. The approach can be generalized to include additional sources of periodic excitation, including sheared currents and depth-dependent magnitude of oncoming current perturbations due to surface gravity waves.

\section{Acknowledgments}

This work was supported by the UK Engineering and Physical Sciences Research Council within Lancaster University Impact Acceleration Account, Grant No. EP/R511560/1. The authors are grateful to SIMEC ATLANTIS Ltd for their advice. All ARCTIC simulations were run on the HEC cluster of Lancaster University.

\section{References}

1. G. Amato, S. Doyle, S. Petley, M.S. Campobasso, I.A. Milne, and G.A. Aggidis. Navier-Stokes CFD Analysis of a Tidal Turbine Rotor in Steady and Planar Oscillatory Flow. In European Wave and Tidal Energy Conference, Cork, Ireland, September 2017.

2. M.S. Campobasso and M.H. Baba-Ahmadi. Analysis of Unsteady Flows Past Horizontal Axis Wind Turbine Airfoils Based on Harmonic Balance Compressible Navier-Stokes Equations with Low-Speed Preconditioning. Journal of Turbomachinery, 134(6), November 2012.

3. M.S. Campobasso, A. Piskopakis, J. Drofelnik, and A. Jackson. Turbulent Navier-Stokes Analysis of an Oscillating Wing in a Power-Extraction Regime Using the Shear Stress Transport Turbulence Model. Computers and Fluids, 88:136-155, December 2013.

4. M.S. Campobasso, M. Yan, A. Bonfiglioli, F.A. Gigante, L. Ferrari, F. Balduzzi, and A.. Bianchini. Low-speed preconditioning for strongly coupled integration of Reynolds-averaged Navier-Stokes equations and two-equation turbulence models. Aerospace Science and Technology, 77:286-298, 2018.

5. A.J. Chorin. A numerical method for solving incompressible viscous flow problems (reprint). Journal of Computational Physics, 135:118-125, 1997.

6. J. Drofelnik, A. Da Ronch, and M.S. Campobasso. Harmonic balance Navier-Stokes aerodynamic analysis of horizontal axis wind turbines in yawed wind. Wind Energy, 21(7):515-530, 2018. DOI: $10.1002 /$ we. 2175 .

7. K.C. Hall, J.P. Thomas, and W.S. Clark. Computations of Unsteady Nonlinear Flows in Cascades Using a Harmonic Balance Technique. AIAA Journal, 40(5):879-886, May 2002.

8. S. Obayashi. Freestream capturing for moving coordinates in three dimensions. AIAA Journal, 30 (4):1125 - 1128, 1992. 